\title{
Improvement of a test-system for detecting inherited and non-inherited genetic changes in living cells
}

\author{
A. Zhuk ${ }^{1,2 *}$, E. Stepchenkova ${ }^{1,2}, \mathrm{~S}$. Inge-Vechtomov ${ }^{1,2}$ \\ ${ }^{1}$ Saint-Petersburg State University, Saint-Petersburg, Russia \\ ${ }^{2}$ Saint-Petersburg Branch of Vavilov Institute of General Genetics, Saint-Petersburg, Russia \\ *e-mail: ania.zhuk@gmail.com
}

Key words: DNA damages, mutations, mutation clusters, genetic safety, alpha-test, genetic toxicology, toxicogenomics

Motivation and Aim: To date, many different tests for evaluating mutagenic activity of chemical, physical and biological factors have been developed and widely used. New dataset of genetic sequence information and new technologies for genetic variation or functional gene expression analysis offer new opportunities for further improvement of classical methods used for evaluating potential environmental toxicants. Here we present an approach that enabling us to increase the efficiency of the alpha-test, an original method which was developed at the Department of Genetics and Biotechnology of the Saint-Petersburg State University.

Methods and Algorithms: Yeast Saccharomyces cerevisiae where used as a model organism. Alpha-test, forward and reverse mutagenesis assays and whole-genome sequencing where used to estimate genome instability. Additionally, we used a wide range of molecular genetic methods (gene cloning, yeast and bacterial cells transformation, polymerase chain reaction, primer and plasmid design methods, chromosome loss induction method), fluorescence microscopy methods, and flow cytometry.

Results: Using the alpha-test we evaluated the spectrum and frequency of inherited and non-inherited changes of genetic material, arising during induction of primary DNA lesions of various types induced by reference mutagens. Using yeast model we investigated distribution of mutations in the genome and the formation of homozygous mutation clusters in diploid yeast cells [1]. Combination of whole-genome sequencing with classical yeast genetics allowed us to investigate initial stage of mutation formation and possible mechanisms of phenotypic changes in non-dividing cells.

Conclusion: An integrated approach that combines methods of classical and molecular genetics, modern molecular genetics, cytological approaches and genome sequencing reveal opportunity for further development of a highly sensitive test system for genetic toxicology, that allows to detect genotoxic factors in eukaryotic cells without using a large number of selective media and manual labor.

Acknowledgements: The reported study was funded by RFBR according to the research project No. 18-34-00130.

\section{References}

1. Lada A.G. et al. (2017) Recombination is responsible for the increased recovery of drug-resistant mutants with hypermutated genomes in resting yeast diploids expressing APOBEC deaminases. Front. Genet. 8:A.202. 